\title{
Cemented hemi-arthroplasty for unstable intertrochanteric fractures in the elderly: a retrospective study
}

\author{
Satya R. Patra' ${ }^{1}$, Naresh K. Panigrahi' ${ }^{1 *}$, Madini Manoj ${ }^{1}$, Saswat Samant ${ }^{2}$, Hemanta K. Bamidi', \\ Kishore C. Das ${ }^{1}$, Siddhartha S. Mohanty ${ }^{1}$
}

${ }^{1}$ Department of Orthopaedics, Hi-Tech Medical College and Hospital, Bhubaneswar, Odisha, India

${ }^{2}$ Department of Orthopaedics, Kalinga Institute of Medical Sciences, Bhubaneswar, Odisha, India

Received: 18 August 2020

Revised: 01 September 2020

Accepted: 02 September 2020

*Correspondence:

Dr. Naresh K. Panigrahi,

E-mail: panigrahi2020naresh@gmail.com

Copyright: (c) the author(s), publisher and licensee Medip Academy. This is an open-access article distributed under the terms of the Creative Commons Attribution Non-Commercial License, which permits unrestricted non-commercial use, distribution, and reproduction in any medium, provided the original work is properly cited.

\begin{abstract}
Background: Unstable intertrochanteric fractures belonging to $31 \mathrm{~A} 2$ and $\mathrm{A} 3$ varieties are difficult challenges for orthopaedic surgeons, particularly in the elderly patients. Osteosynthesis by dynamic hip screw or proximal femoral nail are often plagued by complications like screw cut-out, excessive collapse and fixation failures due to osteoporotic bones. Because of these complications, patients are often kept confined to the bed which may increase the risks of pressure sores, venous thrombosis and pulmonary infections. So, it is desirable to mobilize these elderly patients as quickly as possible following surgery. In recent decades, primary hemi-arthroplasty has emerged as a valuable treatment option for mobilizing these patients early.

Methods: We present our retrospective study on 27 patients above 60 years of age, who were managed with cemented bipolar hemi-arthroplasty after sustaining unstable intertrochanteric fractures. All the patients were operated through modified Hardinge approach. The fractured fragments were secured by stainless steel wiring, particularly the greater trochanter, wherever necessary.

Results: Twenty-three patients $(85 \%)$ were able to stand up with walkers by third post-operative day and were able to walk by fifth post-operative day. There was one case of hip dislocation; it was reduced under anaesthesia. No other complications were encountered at an average follow-up of 3.1 years. The Harris hip score was 'good' or 'excellent' in more than $60 \%$ patients.

Conclusions: Cemented hemi-arthroplasty appears to be a reliable treatment method for unstable intertrochanteric fractures in the elderly and it allows early weight-bearing and rehabilitation in most patients following surgery.
\end{abstract}

Keywords: Intertrochanteric fractures, Unstable hip fractures, Hemi-arthroplasty, Bipolar prosthesis, Fractures in elderly

\section{INTRODUCTION}

Intertrochanteric fractures are the commonest fractures in the elderly patients. ${ }^{1}$ The incidence of these injuries is increasing every year and is expected to be doubled by the year 2040. 2,3 These fractures are often complicated by medical co-morbidities like diabetes, hypertension, osteoporosis, renal diseases and chronic lung diseases making them prone to complications of prolonged confinement to bed, i.e. pressure sores, venous thrombosis, and pulmonary embolism. ${ }^{3,4}$ All these serious risk factors can increase the one-year mortality rate up to $20-30 \%$ in hip fractures. ${ }^{3,5,6}$ Intertrochanteric fractures with posteromedial comminution involving the lesser trochanter, with subtrochanteric extension of fracture and the reverse oblique type fractures are considered unstable varieties. ${ }^{2}$ Almost $50-60 \%$ of trochanteric fractures belong to the unstable group. ${ }^{2}$ These fractures can be classified using the 
AO/OTA classification as 31A1, 31A2 and 31A3 types, and among them A2 and A3 varieties are considered unstable in nature, and as per Evans classification, types III, IV and V are known as unstable fractures. ${ }^{1}$

Various clinical studies have revealed that early rehabilitation and early mobilization out of the bed are beneficial in preventing the complications of confinement to bed as well as mortality. ${ }^{1,7}$ Stable fixation of the fractures and early mobilization is the primary goal of surgical intervention. ${ }^{7-9}$ Evidence also suggest that, surgical interventions within 72 hours of injury can minimize post-operative complications, even mortality rate. ${ }^{1}$ But, early intervention and rehabilitation is often easier said than done, considering the fracture comminution, instability, osteoporosis, poor soft tissue status and underlying medical conditions. ${ }^{8}$

Proximal femoral nails (PFN) and dynamic hip screws (DHS) are widely recommended for internal fixation of intertrochanteric fractures all over the world. ${ }^{3,5}$ But these internal fixation methods are often plagued by failures due to screw cut-out, excessive collapse of fractures, loss of fixation, malunion, and nonunion etc. ${ }^{5,8-10}$ Because of these complications early full-weight bearing mobilization in the elderly with osteoporosis and unstable fracture morphology can be very risky. Therefore, some researchers turn to cemented or uncemented hemiarthroplasty as an option to achieve a stable hip joint suitable for early weight-bearing rehabilitation. . $^{3,5,10-12}$

In the current study, we present our mid-term experience of managing the unstable intertrochanteric fractures in the elderly by cemented bipolar hemiarthroplasty and early rehabilitation.

\section{METHODS}

The study was conducted in the Department of Orthopedics of a tertiary care hospital (Hi-Tech Medical College and Hospital, Bhubaneswar, Odisha, India). It is a retrospective study involving 27 patients in the elderly age-group (>60 years) with unstable intertrochanteric fractures who were treated by cemented hemiarthroplasty surgery using bipolar prostheses during the period from March 2013 to December 2018.

\section{Inclusion criteria}

Patients above 60 years age, AO/OTA 31 A2 and A3 types of intertrochanteric fractures.

\section{Exclusion criteria}

Patients less than 60 years age, previously operated cases, patients with ipsilateral shaft fractures, patients with neurological deficit.

Among the patients 15 were females and 12 were males, and all the patients had good ambulatory capacity before sustaining injury. None of the patients had bilateral injury. Eleven patients $(40.7 \%)$ sustained fractures while riding bikes or bicycles, rest 16 patients $(59.3 \%)$ suffered their injuries due to fall at home. One patient had previous fracture in the contralateral hip, which was operated with a DHS and had resulted in satisfactory functional outcome. One patient had Colle's fracture on the ipsilateral side and was treated with closed reduction and Colle's cast application.

All the patients were operated under spinal anesthesia by a lateral modified Hardinge approach in a lateral decubitus position. Gentamicin impregnated polymethyl methacrylate (PMMA) bone cement was used for all the prostheses (Figure 1). The greater and lesser trochanters were reinforced with stainless steel wiring wherever necessary (Figure 2). Care was taken to prevent leakage of bone cement through the fracture lines. Special precautions were taken to position the stem in desired anteversion as the bony landmarks were disturbed in multiple cases. Vacuum drain was used in all the cases.

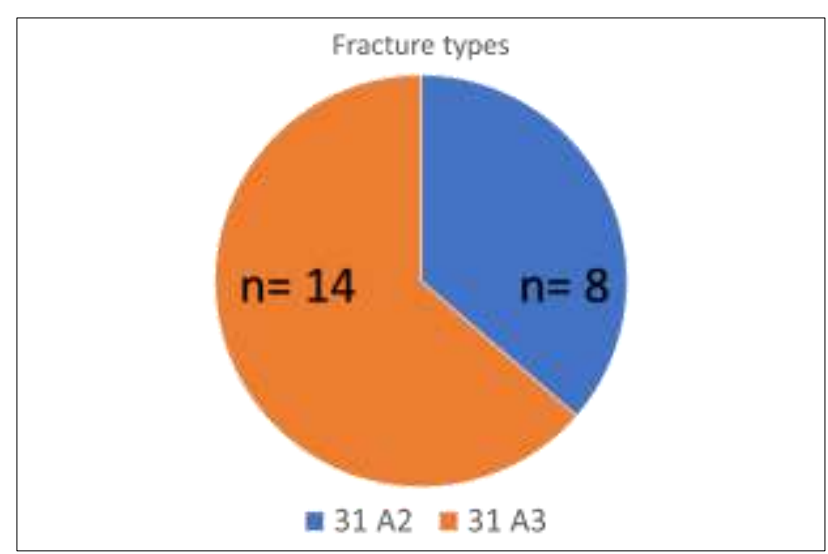

Figure 1: Types of fractures in the study group.

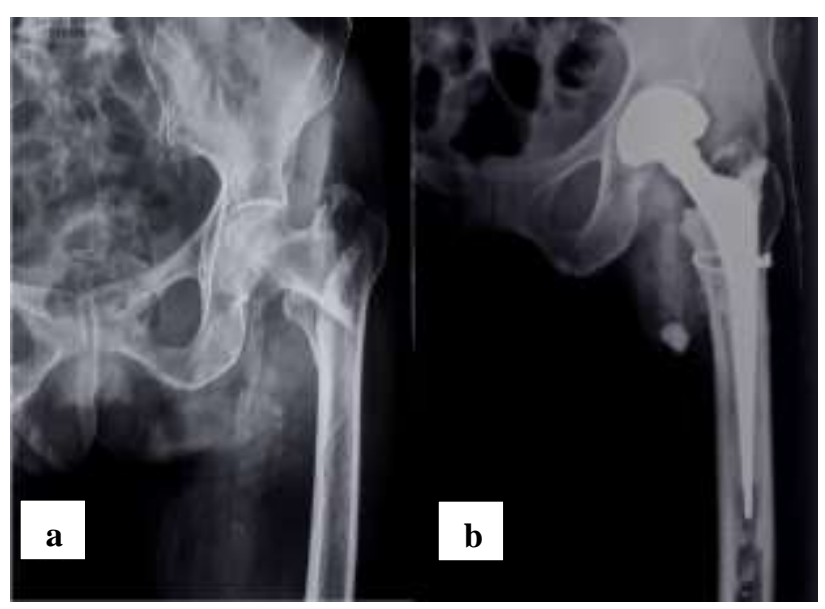

Figure 2: (a) Radiograph showing unstable intertrochanteric fracture of left femur, AO/OTA type 31A2.3; (b) cemented bipolar hemiarthroplasty surgery done with cerclage wiring as seen on postoperative X-ray. 


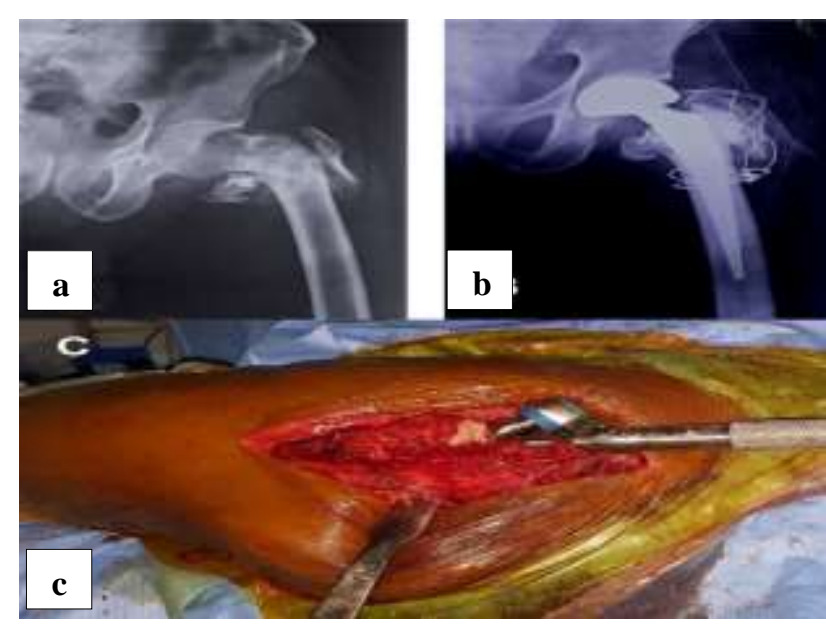

Figure 3: (a) Radiograph showing AO/OTA type

31A2.3 intertrochanteric fracture with gross comminution; (b) x-ray showing cemented bipolar hemiarthroplasty with stainless-steel wiring of the comminuted fragments; (c) per-operative photograph showing the placement of the cemented stem as well as the stainless-steel wiring.

Low molecular weight heparin was used for thromboprophylaxis as a routine in all the patients from the first post-operative day. All the patients were made to stand up with the help of a walker between first and third postoperative day under physiotherapy supervision. Apart from two patients who needed intensive care for three days each due to some respiratory difficulty, there were no other immediate post-operative complications. Seven patients were diabetic and received insulin therapy during the perioperative period. Twenty-three patients were able to walk with the support of walker and physiotherapy supervision by fifth post-operative day; except three patients who could not start walking due to generalized weakness and one patient who had an anterior dislocation of the operated hip after getting discharged on the eighth post-operative day. The dislocation was reduced by closed manner under spinal anesthesia and fluoroscopy control.

Patients were followed up at 2 weeks, 4 weeks, 6 weeks, 3 months and subsequently every three months. Standard antero-posterior radiographs of the pelvis and both hip joints including the upper half of thighs in internal rotation were routinely done for radiological evaluation along with lateral radiographs of the operated hips. The modified Harris hip score (mHHS) was used for assessment of functional recovery. ${ }^{13}$

\section{RESULTS}

Two patients expired (3rd and 5th months) due to other medical conditions and three were lost to follow-up; therefore, 22 patients were retrospectively evaluated for clinical, radiological and functional outcomes.

The average age of the patients was 71.7 years (range, 6089 years) (Figure 4 ). The mean duration of surgery from incision to closure was found to be 91.4 minutes (range, 76-210 minutes). The mean blood loss was recorded as 235 cc (range, 150- $750 \mathrm{cc}$ ). Average duration of the hospital stay is 7.3 days (range, 5-23 days). All patients had good wound healing with stitch removal at 2 weeks' time, except two patients who had delayed healing and stitches were remove after 3 weeks. All patients except two were able to stand up with the help of a walker by the third postoperative day and were able to start full-weight-bearing walking with support by the fifth post-operative day.

Only one patient suffered dislocation of the hip on the eighth post-operative day; there were no incidence of infection, implant loosening, deep vein thrombosis (DVT), pulmonary embolism, pressure sores and lung atelectasis. None of the patients needed re-surgery till the last follow up in December 2018. Only two patients (9\%) showed evidence of slight subsidence of the implant on radiographs, but there were no appreciable clinical consequences.

The mHHS were calculated for all the 22 patients at twoand three-years follow-up (Figure 5). At the end of two years, eight patients $(36 \%)$ had 'fair' outcome, 11 patients (50\%) had 'good' and three patients (14\%) had excellent outcome. At the end of third year follow up, two patients from the 'fair' category improved functionally to be included in the 'good' category.

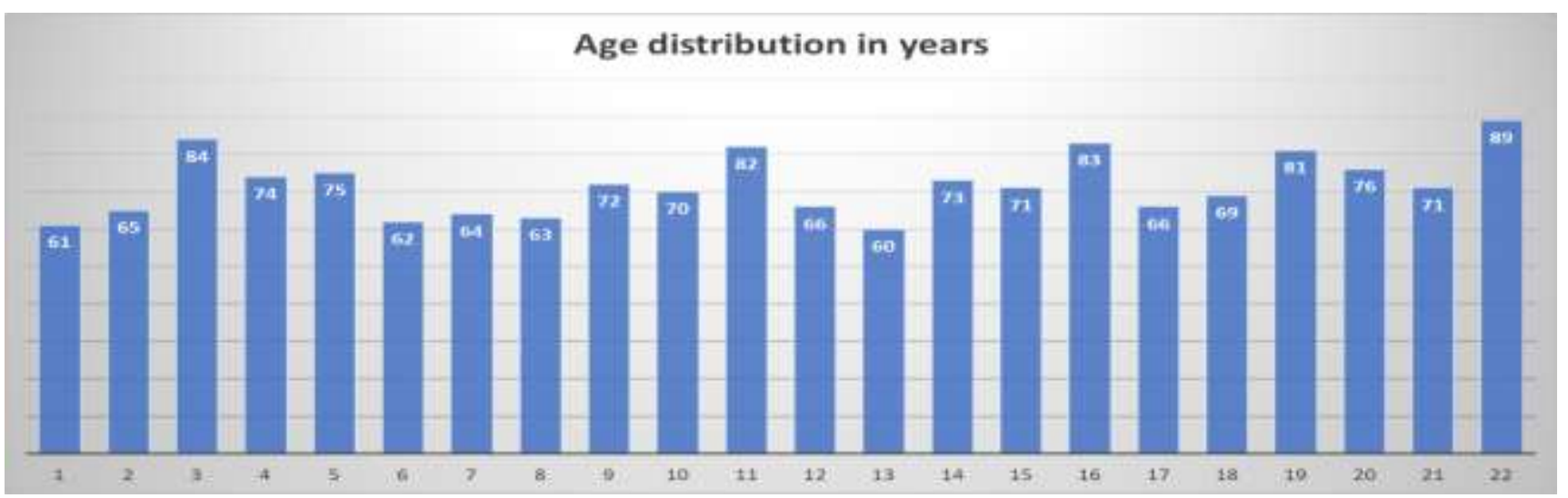

Figure 4: Age distribution of the patients in the study group. 


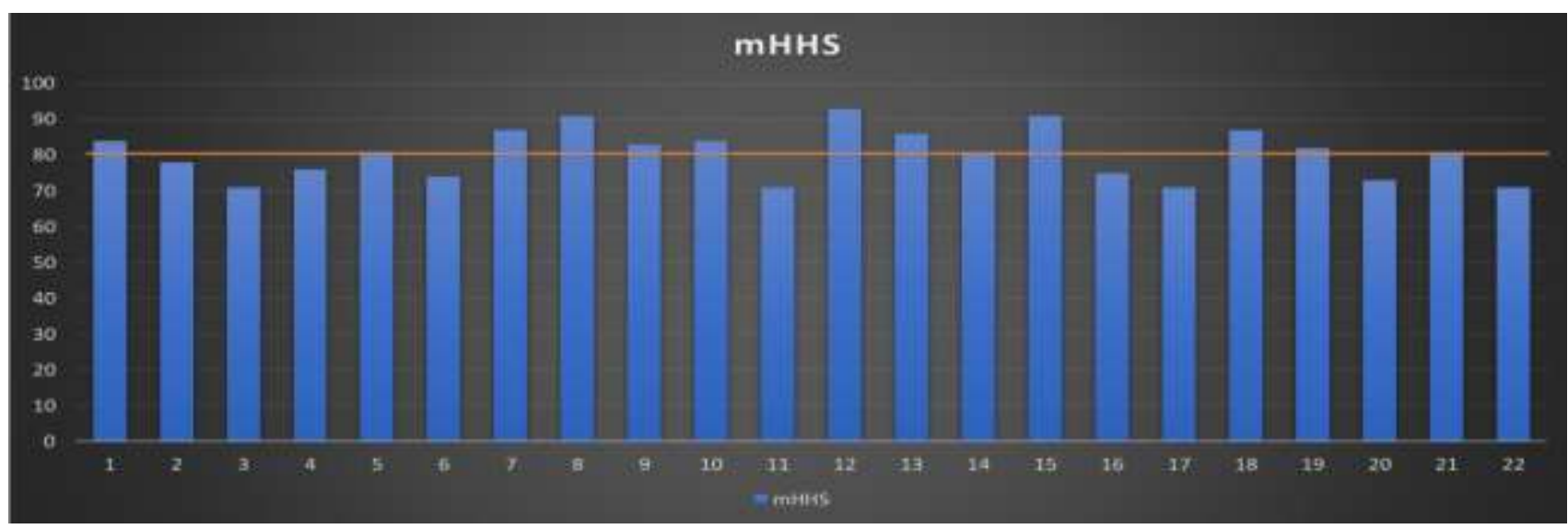

Figure 5: Distribution of mHHS values among the study group.

The orange line depicting the mean mHHS of 80.5 (range, 71-93).

\section{DISCUSSION}

Unstable intertrochanteric fractures belonging to types A2 and A3 are challenging problems, particularly in the elderly age-group of $>60$ years. Various medical comorbidities prevalent in the elderly such as osteoporosis, diabetes, hypertension, cardiac diseases, renal compromise, chronic lung diseases, obesity, dementia and eye-sight problems. render the management prone to complications. Although internal fixation by PFN or proximal femoral nail anti-rotation (PFNA) has been accepted as standard surgical treatment for the intertrochanteric fractures, their success rate is notably inferior in the osteoporotic bones in the elderly. ${ }^{5,8,9}$ After internal fixation of these unstable fractures weight bearing and ambulation is always delayed in the fear of screw cutout and loss of fixation. Therefore, hemiarthroplasty is emerging as a viable alternative to internal fixation in recent decades. ${ }^{1-6,8,10-12}$

In this study, we retrospectively followed up 22 elderly patients of unstable intertrochanteric fractures treated by cemented bipolar hemiarthroplasty as the primary management plan. We observed that, hemiarthroplasty in the unstable fracture scenario is more challenging than the routine hemiarthroplasty surgeries for fractures of the neck of femur. Reduction of the fracture fragments, cementing without spillage, placement of the stem inside the unstable proximal femur, reattachment of the greater trochanter with the shaft, and reinforcement with cerclage wires; all these steps make this surgery technically more demanding. The average duration of the surgery in our series was 91.4 minutes. Jolly et al reported an average duration on 60.4 minutes, Kumar et al reported a mean duration of 116 minutes, 106 minutes by Mansukhani et al, 96 minutes by Thakur et al and a mean of 77.5 minutes by Zhou et al. ${ }^{2,3,8-}$ 10 The average intra-operative blood loss and average hospital stay in our patients were similar to the other studies those we reviewed.

Due to the comminuted nature of the fractures, the balance of the hip joint is also disturbed and difficult to recreate.
We had one case of dislocation (4\%) in our series. Jolly et al reported a dislocation rate of $10 \%$ in a study group of 50; Celiktas et al had just one dislocation in a series of 64 patients, whereas Mansukhani et al encountered two dislocation (15\%) among 13 patients. ${ }^{2,4,9}$ These findings indicate that it is important to obtain good soft tissue balance by careful repair in these cases during prosthesis implantation. Minimum damage to the surrounding tissues during dissection can also prove very crucial for postoperative hip stability.

There were no other significant peri-operative complications like superficial or deep infections; only two patients showed signs of delayed wound healing. No incidence of pressure sores, urinary tract infections, deep vein thrombosis or pulmonary embolism was seen. After longer follow up (mean of 3.1 years), there were no periprosthetic fractures, implant loosening or acetabular arthritic changes.

Eighteen patients could walk bearing full weight with the help of a walker by the end of third post-operative day; rest four patients took around three to four weeks to walk on their own. In most of the studies, patients of hemiarthroplasty started weight-bearing ambulation with support by the second post-operative day. Patients of this age-group could not comprehend partial weight bearing concept using walker; therefore, in most of the studies including ours, full weight-bearing was the norm and patients tolerated it very well following cemented hemiarthroplasty. The opinion in the literature is more in favor of hemiarthroplasty being helpful in immediate mobilization of the patients after surgery, and the same may not always be achieved after internal fixation of such fractures. But good reduction and stabilization of the postero-medial fragment is important for prevention of subsidence. ${ }^{12}$

On functional evaluation by the mHHS, 14 patients $(64 \%)$ had good or excellent result at minimum follow-up of three years. Even the patient who had dislocation scored 73 in mHHS to be included in the 'fair' category at three years' 
follow-up. Kumar et al had shown an average score of 75 (fair) in their series at one year follow up; Singh et al reported 'fair to good' score in all their patients at the end of one year, Kim et al observed 'good' score in most of their patients of uncemented hemiarthroplasty, whereas Zhou et al had 'good' score in most of their patients of cemented bipolar hemiarthroplasty at the end of first year. , $6,8,12$ Among the literatures we searched and cited, our series appears to have a comparatively longer followup of at least three years for functional evaluation.

Our study has certain limitations such as, small sample size, lack of a control group or comparison with PFN, lack of long duration of follow-up and being retrospective in nature.

\section{CONCLUSION}

With this mid-term range of follow-up and retrospective analysis we conclude that, cemented bipolar hemiarthroplasty for unstable osteoporotic intertrochanteric fractures in the elderly patients above 60 years of age is a reliable alternative treatment option to osteosynthesis. It recreates a stable hip joint that can withstand the forces around it and also allows for immediate post-operative weight-bearing ambulation in patients those may lack the compliance towards nonweight-bearing or partial weight-bearing rehabilitation due to age related factors. Minimum dissection and meticulous repair of the soft-tissue around the hip as well as cerclage wiring of the major fragments is crucial for satisfactory outcome and long-term survival of the joint. Prospective comparative study with other modalities of management is essential to determine the most effective surgical option for these complicated injuries.

Funding: No funding sources

Conflict of interest: None declared

Ethical approval: The study was approved by the institutional ethics committee

\section{REFERENCES}

1. Ju J, Zhang P, Jiang B. Hip replacement as alternative to intramedullary nail in elderly patients with unstable intertrochanteric fractures: A systematic review and meta-analysis. Orthop Surg. 2019;11:745-54.

2. Mansukhani SA, Tuteja SV, Kasodekar VB, Mukhi SR. A comparative study of the dynamic hip screw, the cemented bipolar hemiarthroplasty and the proximal femoral nail for the treatment of unstable intertrochanteric fractures. J Clin Diagn Res. 2017;11(4):14-9.

3. Zhou S, Liu J, Zhen P, Shen W, Chang Y, Zhang H, Zhu Q, Li X. Proximal femoral nail anti-rotation versus cemented bipolar hemiarthroplasty for unstable femoral intertrochanteric fracture in the elderly: A retrospective study. BMC Musculoskelet Disord. 2019;20:500.

4. Celiktas M, Togrul E, Kose O. Calcar preservation arthroplasty for unstable intertrochanteric femoral fractures. Clin Orthop Surg. 2015;7:436-42.

5. Ucpunar H, Camurcu Y, Cobden A, Sofu H, Kis M, Demirel H. Comparative evaluation of postoperative health status and functional outcome in patients treated with either proximal femoral nail or hemiarthroplasty for unstable intertrochanteric fractures. J Orthop Surg. 2019;27(3):1-9.

6. Singh S, Shrivastava C, Kumar S. Hemi replacement arthroplasty for unstable intertrochanteric fractures of femur. J Clin Diagn Res. 2014;8(10):1-4.

7. Yoo JI, Ha YC, Lim JY, Kang H, Yoon BH, Kim H. Early rehabilitation in elderly after arthroplasty versus internal fixation for unstable intertrochanteric fractures of femur: a systematic review and metaanalysis. J Korean Med Sci. 2017;32:858-67.

8. Kumar Gn K, Meena S, Kumar VN, Manjunath S, Raj Mk V. Bipolar hemiarthroplasty in unstable intertrochanteric fractures in elderly: A prospective study. J Clin Diagn Res. 2013;7(8):1669-71.

9. Jolly A, Bansal R, More AR, Pagadala MB. Comparison of complications and functional results of unstable intertrochanteric fractures of femur treated with proximal femur nails and cemented hemiarthroplasty. J Clin Orthop Trauma. 2019;10:296-301.

10. Thakur A, Lal M. Cemented hemiarthroplasty in elderly osteoporotic unstable intertrochanteric fractures using fracture window. Malays Orthop J. 2016;10(1):5-10.

11. Lee YK, Park CH, Koo KH. Fixation of trochanteric fragments in cementless bipolar hemiarthroplasty of unstable intertrochanteric fractures: cerclage wiring. Hip Pelvis. 2017;29(4):262-9.

12. Kim Y, Moon JK, Hwang KT, Choi IY, Kim YH. Cementless bipolar hemiarthroplasty for unstable intertrochanteric fractures in octogenarians. Acta Orthop Traumatol Turc. 2014;48(4):424-30.

13. Vishwanathan K, Akbari K, Patel AJ. Is the modified Harris hip score valid and responsive instrument for outcome assessment in the Indian population with pertrochanteric fractures? J Orthop. 2018;15(1):40-6.

Cite this article as: Patra SR, Panigrahi NK, Manoj M, Samant S, Bamidi HK, Das KC, Mohanty SS. Cemented hemi-arthroplasty for unstable intertrochanteric fractures in the elderly: a retrospective study. Int J Res Orthop 2020;6:1166-70. 